\title{
Polyphenolic profiles in edible Annona spp. using high-performance liquid chromatography (HPLC-MS/MS)
}

\author{
Shetty M. J. $\mathbf{1}^{1}, \quad$ Vasudeva K. $\mathbf{R}^{1}$, Sakthivel $T^{2}$, Suresh G. J. ${ }^{3}$, Krishna H. C. ${ }^{4}$, \\ Vishnuvardhana ${ }^{5}$ and Kumar A. $S^{6}$
}

Received: 22.10.2020

Revised: 25.11 .2020

Accepted: 01.12.2020

\begin{abstract}
Polyphenolic compounds of fruits of various plant species play an important role in physiological functions related to human health. Polyphenols have important biological activities like antioxidant activity which helps in normal functioning of human body. The objective of this study was to investigate the profiles of polyphenolic compounds in different edible Annona spp. fruits. Total of eighteen identified individual phenolic compounds were found, among which p-coumaric acid (321.53-90.17 $\left.\mu \mathrm{g} \mathrm{g}^{-1} \mathrm{FW}\right)$, o-coumaric acid $\left(70.80-19.00 \mu \mathrm{g} \mathrm{g}^{-1} \mathrm{FW}\right)$, 2,4-dihydroxybenzoic acid (39.49$\left.10.43 \mu \mathrm{g} \mathrm{g}^{-1} \mathrm{FW}\right)$, caffeic acid $\left(35.26-3.43 \mu \mathrm{g} \mathrm{g}^{-1} \mathrm{FW}\right)$, gentisic acid (24.69-10.46 $\left.\mu \mathrm{g} \mathrm{g}^{-1} \mathrm{FW}\right)$, protocatechuic acid (17.04-4.23 $\mu \mathrm{g}$ $\left.\mathrm{g}^{-1} \mathrm{FW}\right), t$-cinnamic acid (22.68-3.93 $\left.\mu \mathrm{g} \mathrm{g}^{-1} \mathrm{FW}\right)$ and ferulic acid (21.78-3.43 $\left.\mu \mathrm{g} \mathrm{g}^{-1} \mathrm{FW}\right)$ were abundant in annona fruits while benzoic acid (23.28-4.61 $\left.\mu \mathrm{g} \mathrm{g}^{-1} \mathrm{FW}\right)$, $p$-hydroxybenzoic acid $\left(1.79-0.31 \mu \mathrm{g} \mathrm{g}^{-1} \mathrm{FW}\right)$, salicylic acid $\left(6.00-2.40 \mu \mathrm{g} \mathrm{g}^{-1} \mathrm{FW}\right), 3-$ hydroxybenzoic acid (6.05-0.88 $\left.\mu \mathrm{g} \mathrm{g}^{-1} \mathrm{FW}\right)$, vanillic acid. (19.13-2.16 $\left.\mu \mathrm{g} \mathrm{g}^{-1} \mathrm{FW}\right)$, gallic acid (15.88-2.74 $\left.\mu \mathrm{g} \mathrm{g}^{-1} \mathrm{FW}\right)$, ellagic acid (1.12-0.20 $\left.\mu \mathrm{g} \mathrm{g}^{-1} \mathrm{FW}\right)$, syringic acid $\left(0.78-0.34 \mu \mathrm{g} \mathrm{g}^{-1} \mathrm{FW}\right)$ and sinapic acid (2.16-0.79 $\left.\mu \mathrm{g} \mathrm{g}^{-1} \mathrm{FW}\right)$ were limited. However, chlorogenic acid was not detected. The results obtained in this study will furnish a better knowledge of the polyphenolic composition in annona fruits.
\end{abstract}

Key Words: Annona spp., HPLC-MS/MS, o-coumaric acid, p-coumaric acid, polyphenols

\section{Introduction}

Biological properties of polyphenolic compounds like antioxidant activities are essential to prevent many diseases including cancer, rheumatoid arthritis, cardio vascular and diseases related to degenerative process (Almeida et al., 2011; Dembitsky et al., 2011). Several studies have shown high correlation between the consumption of fruits in prevention and treatment of various diseases (Loizzo et al., 2012). The genus Annona belongs to the annonaceous family and comprises of approximately 162 species of trees and shrubs (Chatrou et al., 2012). Economically, this genus is the most important of the annonaceae family due to its edible fruits and medicinal properties. While, there has been no comparative study on the biochemical properties of these fruits (Sousa et al., 2012). In this paper, a comprehensive study on the

Author's Address

${ }^{1}$ Department of PHT, College of Horticulture, Bengaluru

${ }^{2}$ Division of Fruit Crops, IIHR, Bengaluru

${ }^{3}$ Department of PHT, College of Horticulture, Bengaluru

${ }^{4}$ Department of PHT, College of Horticulture, Kolar

${ }^{5}$ Regional Horticulture Experimental Research Station, Bengaluru

${ }^{6}$ Krishi Vigyan Kendra, Kolar

E-mail.: jshettymanju@gmail.com polyphenolic profiles of eight different annona genotype using an HPLC method with a TQD (Triple Quadrupole) mass spectrometry is reported.

\section{Material and Methods}

Fresh annona fruits used for experimentation were procured from the orchards of Bioversity International, Indian Institute of Horticultural Research (IIHR) and Regional Horticulture Research and Extension Centre (RHREC), Bengaluru. Ripened fruits of uniform size, shape and maturity, free from visible damages were procured and carefully placed in corrugated fibre board boxes and brought to laboratory.

The individual polyphenolic acids for High performance liquid chromatography-tandem mass spectrometry (HPLC-MS/MS) (Acquity Class-H system, Waters, Burnsville, USA) analysis were isolated using 80 per cent methanol (Weidner et al., 2000). 10 grams of Annona fruit pulp was homogenized in methanol $(80 \%)$, centrifuged and volume was made up to $50 \mathrm{~mL}$. $20 \mathrm{~mL}$ of extract was evaporated near to dryness under the vacuum at $45^{\circ} \mathrm{C}$ and then diluted to $5 \mathrm{~mL}$ with water. Later 
extracted thrice with petroleum ether and then in 40 $\mathrm{mL}$ of ethyl acetate using separating funnel. Aqueous layer formed was discarded; ethyl acetate extract was evaporated to dryness under vacuum at room temperature. To the dry residue, $4 \mathrm{~mL}$ of $2 \mathrm{~N}$ $\mathrm{NaOH}$ was added and allowed overnight for hydrolyzing. Extract was acidified to $\mathrm{pH} 2$ by using $5 \mathrm{~mL} 2 \mathrm{~N} \mathrm{HCl}$, again re-extracted with $50 \mathrm{~mL}$ ethyl acetate. Ethyl acetate layer was again re-extracted twice with $25 \mathrm{~mL}$ of $0.1 \mathrm{~N} \mathrm{NaHCO}_{3}$. The aqueous layer was further acidified to $\mathrm{pH} 2$ with $5 \mathrm{~mL} 2 \mathrm{~N}$ $\mathrm{HCl}$ and extracted thrice with $25 \mathrm{~mL}$ ethyl acetate, the ethyl acetate layer was dried completely in rotary evaporator and the residue was dissolved in 2 $\mathrm{mL}$ MS grade methanol, filtered through $0.2 \mu \mathrm{m}$ nylon filter prior to injection into HPLC-MS/MS system (Acquity Class-H system, Waters, Burnsville, USA) for polyphenolic acid estimation.

Table 1. Treatment details

\begin{tabular}{|l|l|}
\hline SN & Species / Varieties used in the study \\
\hline 1. & Annona squamosa L.(Sweetsop) \\
\hline a. & Balanagar \\
\hline b. & Arka Sahan \\
\hline c. & Red Sitaphal \\
\hline 2. & Annona atemoya Hort. \\
\hline 3. & Annona reticulata L. (Ramphal) \\
\hline 4. & Annona muricata L.(Soursop) \\
\hline 5. & Annona cherimola M. (Cherimoya) \\
\hline 6. & Annona glabra L. (Pond apple) \\
\hline
\end{tabular}

\section{LC and MS-MS conditions}

The polyphenolic acids were resolved on the analytical column BEH-C18 $(2.1 \times 50 \mathrm{~mm}, 1.7 \mu \mathrm{m})$ from waters India Ltd., protected by a Vanguard BEH C-18 (Waters, USA) with the gradient flow of organic and aqueous phase with the flow rate of 0.3 $\mathrm{mL}$ minute $^{-1}$. The column temperature was maintained at $25^{\circ} \mathrm{C}$ during analysis and the sample injection volume was $2 \mu \mathrm{L}$. The eluted polyphenolic acids were monitored by a PDA detector and the UPLC column effluent pumped directly without any split into the TQD-MS/MS (Waters, USA) system optimized for the polyphenolic acid analysis.

\section{Mobile phase}

Solvent - A: 0.1 per cent formic acid in water and Solvent - B: 0.2 per cent formic acid in methanol

\section{Statistical analysis}

Completely Randomized Design (CRD) and mean values were compared using Duncan's Multiple Range Test (DMRT).

\section{Results and Discussion}

Total seventeen individual polyphenolic acids were fractioned and quantified from the methanolic extracts of fruit pulp of Annona spp. by HPLCMS/MS analysis. It is apparent from mean values that polyphenolic acids viz., $p$-coumaric acid, ocoumaric acid, 2,4-dihydroxybenzoic acid, caffeic acid, gentisic acid, protocatechuic acid, $t$-cinnamic acid and ferulic acid were abundant in annona fruits while benzoic acid, $p$-hydroxybenzoic acid, salicylic acid, 3-hydroxybenzoic acid, vanillic acid, gallic acid, ellagic acid, syringic acid and sinapic acid were limited (Figure 1a and 1b). However, chlorogenic acid was not detected. The polyphenolic acid content was significantly different among the annona genotypes and is depicted in Table 2.

There was a significant difference for benzoic acid among the annona genotypes. A range of 3.63 to $23.28 \mu \mathrm{g} \mathrm{g}^{-1} \mathrm{FW}$ of benzoic acid was recorded in different genotypes. As evident from the treatment means, maximum benzoic acid was found in $A$. muricata $\left(23.28 \mu \mathrm{g} \mathrm{g}^{-1} \mathrm{FW}\right)$ which was followed by A. reticulata $\left(13.69 \mu \mathrm{g} \mathrm{g}^{-1} \mathrm{FW}\right)$, Balanagar $(10.02 \mu \mathrm{g}$

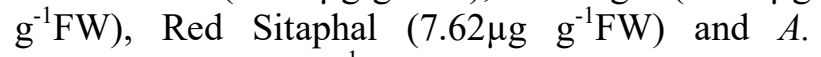
cherimola $\left(6.79 \mu \mathrm{g} \mathrm{g}^{-1} \mathrm{FW}\right)$. The minimum benzoic acid was found in A. atemoya $\left(3.63 \mu \mathrm{g} \mathrm{g}^{-1} \mathrm{FW}\right), A$. glabra $\left(4.61 \mu \mathrm{g} \mathrm{g}^{-1} \mathrm{FW}\right)$ and Arka Sahan $\left(6.09 \mu \mathrm{g} \mathrm{g}^{-}\right.$ $\left.{ }^{1} \mathrm{FW}\right)$. The $p$-hydroxybenzoic acid was significantly different among the annona genotypes. phydroxybenzoic acid ranged from 0.31 to $1.97 \mu \mathrm{g} \mathrm{g}^{-}$ ${ }^{1} \mathrm{FW}$ in different genotypes. As evident from treatment means, the maximum $p$-hydroxybenzoic acid was noticed in Arka Sahan $\left(1.97 \mu \mathrm{g} \mathrm{g}^{-1} \mathrm{FW}\right)$ which showed statistically $(p<0.05)$ par with Red Sitaphal $\left(1.79 \mu \mathrm{g} \mathrm{g}^{-1} \mathrm{FW}\right)$, was followed by $A$. reticulata $\left(1.02 \mu \mathrm{g} \mathrm{g}^{-1} \mathrm{FW}\right)$, A. glabra $(0.61 \mu \mathrm{g} \mathrm{g}$ $\left.{ }^{1} \mathrm{FW}\right)$, A.muricata $\left(0.67 \mu \mathrm{g} \mathrm{g}{ }^{-1} \mathrm{FW}\right)$ and Balanagar $\left(0.58 \mu \mathrm{g} \mathrm{g}^{-1} \mathrm{FW}\right)$. The minimum $p$-hydroxybenzoic acid was observed in A. atemoya $\left(0.31 \mu \mathrm{g} \mathrm{g}^{-1} \mathrm{FW}\right)$ and $A$. cherimola $\left(0.45 \mu \mathrm{g} \mathrm{g}^{-1} \mathrm{FW}\right)$. The salicylic acid was significantly different among the annona genotypes. Salicylic acid ranged from 2.40 to 6.00 $\mu \mathrm{g} \mathrm{g}^{-1} \mathrm{FW}$ in different genotypes. The maximum 
Polyphenolic profiles in edible Annona spp.

Table 2. Phenolic acid ( $\mu \mathrm{g} \mathrm{g}^{-1} \mathrm{FW}$ ) profiling of the selected edible Annona spp. fruits by HPLC-MS/MS

\begin{tabular}{|c|c|c|c|c|c|c|c|c|c|c|c|c|c|c|c|c|c|c|}
\hline 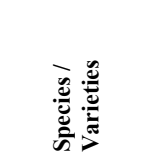 & 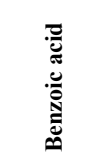 & 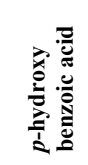 & 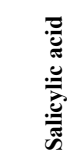 & 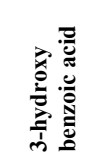 & 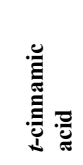 & 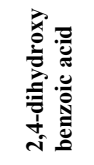 & 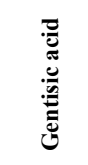 & 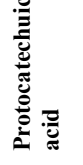 & 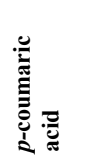 & 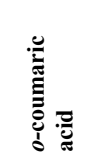 & 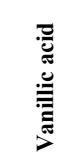 & 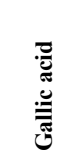 & 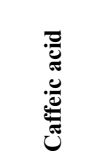 & 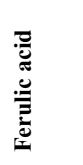 & 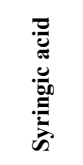 & 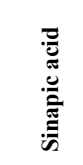 & 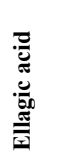 & 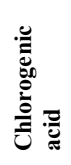 \\
\hline $\begin{array}{l}\text { Annona } \\
\text { squamosa } \\
\text { a) Balanagar }\end{array}$ & $10.02^{\mathrm{c}}$ & $0.58^{\mathrm{cd}}$ & $2.42^{\mathrm{d}}$ & $2.89^{\mathrm{b}}$ & $18.29^{\mathrm{b}}$ & $12.39^{\mathrm{cd}}$ & $18.16^{\mathrm{b}}$ & $4.23^{\mathrm{b}}$ & $115.38^{\mathrm{de}}$ & $48.11^{\mathrm{b}}$ & $4.96^{\mathrm{bc}}$ & $10.25^{\mathrm{b}}$ & $6.31^{\mathrm{f}}$ & $5.93^{\mathrm{d}}$ & $0.77^{\mathrm{a}}$ & $2.16^{\mathrm{a}}$ & $0.28^{\mathrm{cd}}$ & ND \\
\hline $\begin{array}{l}\text { b) Arka } \\
\text { Sahan }\end{array}$ & $6.09^{\mathrm{de}}$ & $1.97^{\mathrm{a}}$ & $4.69^{\mathrm{b}}$ & $1.35^{\mathrm{cd}}$ & $22.68^{\mathrm{a}}$ & $16.31^{\mathrm{cd}}$ & $14.22^{\mathrm{bc}}$ & $14.33^{\mathrm{a}}$ & $90.17^{\mathrm{fg}}$ & $19.00^{\mathrm{de}}$ & $3.11^{\mathrm{cd}}$ & $14.06^{\mathrm{a}}$ & $23.88^{\mathrm{c}}$ & $6.22^{\mathrm{d}}$ & $0.34^{\mathrm{b}}$ & $1.11^{\mathrm{cd}}$ & $0.27^{\mathrm{cd}}$ & ND \\
\hline $\begin{array}{l}\text { c) Red } \\
\text { Sitaphal }\end{array}$ & $7.62^{\mathrm{d}}$ & $1.79^{\mathrm{a}}$ & $2.69^{\mathrm{cd}}$ & $2.92^{\mathrm{b}}$ & $17.54^{\mathrm{b}}$ & $10.43^{\mathrm{d}}$ & $14.84^{\mathrm{bc}}$ & $4.82^{\mathrm{b}}$ & $103.76^{\mathrm{ef}}$ & $36.23^{\mathrm{bc}}$ & $4.10^{\mathrm{cd}}$ & $6.57^{\mathrm{c}}$ & $3.43^{\mathrm{f}}$ & $5.91^{\mathrm{d}}$ & $0.78^{\mathrm{a}}$ & $0.87^{\mathrm{cd}}$ & $0.20^{\mathrm{d}}$ & ND \\
\hline $\begin{array}{l}\text { Annona } \\
\text { atemoya }\end{array}$ & $3.63^{\mathrm{f}}$ & $0.31^{\mathrm{d}}$ & $4.73^{\mathrm{ab}}$ & $1.32^{\mathrm{cd}}$ & $14.07^{\mathrm{c}}$ & $14.83^{\mathrm{cd}}$ & $9.77^{\mathrm{c}}$ & $15.82^{\mathrm{a}}$ & $78.54^{\mathrm{g}}$ & $7.91^{\mathrm{e}}$ & $2.59^{\mathrm{cd}}$ & $8.34^{\mathrm{bc}}$ & $10.07^{\mathrm{e}}$ & $7.24^{\mathrm{d}}$ & $0.75^{\mathrm{a}}$ & $0.86^{\mathrm{cd}}$ & $0.27^{\mathrm{cd}}$ & ND \\
\hline $\begin{array}{l}\text { Annona } \\
\text { reticulata }\end{array}$ & $13.69^{\mathrm{b}}$ & $1.02^{\mathrm{b}}$ & $6.00^{\mathrm{a}}$ & $6.06^{\mathrm{a}}$ & $10.20^{\mathrm{d}}$ & $39.49^{\mathrm{a}}$ & $17.38^{\mathrm{b}}$ & $6.99^{\mathrm{b}}$ & $172.65^{\mathrm{c}}$ & $48.64^{\mathrm{b}}$ & $3.71^{\mathrm{cd}}$ & $15.88^{\mathrm{a}}$ & $25.59^{\mathrm{bc}}$ & $6.13^{\mathrm{d}}$ & $0.38^{\mathrm{b}}$ & $0.79^{\mathrm{d}}$ & $0.40^{\mathrm{c}}$ & ND \\
\hline $\begin{array}{l}\text { Annona } \\
\text { muricata }\end{array}$ & $23.28^{\mathrm{a}}$ & $0.67^{\mathrm{c}}$ & $5.12^{\mathrm{ab}}$ & $3.13^{\mathrm{b}}$ & $4.47^{\mathrm{e}}$ & $27.84^{\mathrm{b}}$ & $24.69^{\mathrm{a}}$ & $17.04^{\mathrm{a}}$ & $321.53^{\mathrm{a}}$ & $70.80^{\mathrm{a}}$ & $2.16^{\mathrm{d}}$ & $6.03^{\mathrm{c}}$ & $16.76^{\mathrm{d}}$ & $15.94^{\mathrm{b}}$ & $0.36^{\mathrm{b}}$ & $1.67^{\mathrm{b}}$ & $0.69^{\mathrm{b}}$ & ND \\
\hline $\begin{array}{l}\text { Annona } \\
\text { cherimola }\end{array}$ & $6.79^{\mathrm{d}}$ & $0.45^{\mathrm{cd}}$ & $2.40^{\mathrm{d}}$ & $0.89^{\mathrm{d}}$ & $3.93^{\mathrm{e}}$ & $18.38^{\mathrm{c}}$ & $11.82^{\mathrm{c}}$ & $16.47^{\mathrm{a}}$ & $260.66^{\mathrm{b}}$ & $23.46^{\mathrm{cd}}$ & $6.87^{\mathrm{b}}$ & $6.35^{\mathrm{c}}$ & $35.26^{\mathrm{a}}$ & $21.78^{\mathrm{a}}$ & $0.67^{\mathrm{a}}$ & $1.26^{\mathrm{bc}}$ & $1.12^{\mathrm{a}}$ & ND \\
\hline $\begin{array}{l}\text { Annona } \\
\text { glabra }\end{array}$ & $4.61^{\mathrm{ef}}$ & $0.61^{\mathrm{c}}$ & $3.97^{\mathrm{bc}}$ & $1.81^{\mathrm{c}}$ & $3.95^{\mathrm{e}}$ & $30.87^{\mathrm{b}}$ & $10.46^{\mathrm{c}}$ & $16.56^{\mathrm{a}}$ & $124.05^{\mathrm{d}}$ & $50.31^{\mathrm{b}}$ & $19.13^{\mathrm{a}}$ & $2.74^{\mathrm{d}}$ & $27.78^{b}$ & $12.28^{\mathrm{c}}$ & $0.63^{\mathrm{a}}$ & $0.99^{\mathrm{cd}}$ & $0.26^{\mathrm{cd}}$ & ND \\
\hline Mean & 9.47 & 0.92 & 4.00 & 2.54 & 11.89 & 21.32 & 15.17 & 12.03 & 158.34 & 38.06 & 5.83 & 8.78 & 18.63 & 10.18 & 0.58 & 1.21 & 0.42 & -- \\
\hline S. Em ( $( \pm)$ & 0.518 & 0.096 & 0.448 & 0.301 & 1.068 & 2.228 & 1.778 & 1.362 & 5.485 & 4.793 & 0.839 & 0.777 & 1.110 & 0.839 & 0.055 & 0.157 & 0.0560 & -- \\
\hline CD (0.05) & 1.553 & 0.289 & 1.345 & 0.903 & 3.203 & 6.681 & 5.331 & 4.085 & 16.445 & 14.371 & 2.515 & 2.331 & 3.329 & 2.518 & 0.166 & 0.470 & 0.167 & -- \\
\hline
\end{tabular}

Note: Values represent means of triplicate readings, values with different superscripts in the same column are significantly different at 95\% level of confidence based on Duncan's multiple-range test 


\section{Shetty et al.}

salicylic acid was found in $A$. reticulata $(6.00 \mu \mathrm{g} \mathrm{g}$ $\left.{ }^{1} \mathrm{FW}\right)$ which was followed by $A$. muricata $(5.12 \mu \mathrm{g}$ $\left.\mathrm{g}^{-1} \mathrm{FW}\right)$, A. atemoya $\left(4.73 \mu \mathrm{g} \mathrm{g}^{-1} \mathrm{FW}\right)$, Arka Sahan ( $\left.4.69 \mu \mathrm{g} \mathrm{g}^{-1} \mathrm{FW}\right)$ and $A$. glabra $\left(3.97 \mu \mathrm{g} \mathrm{g}^{-1} \mathrm{FW}\right)$. The minimum salicylic acid was found in and $A$. cherimola $\left(2.40 \mu \mathrm{g} \mathrm{g}^{-1} \mathrm{FW}\right)$, Balanagar $(2.42 \mu \mathrm{g} \mathrm{g}$ $\left.{ }^{1} \mathrm{FW}\right)$ and Red Sitaphal $\left(2.69 \mu \mathrm{g} \mathrm{g}^{-1} \mathrm{FW}\right)$. Annona genotypes exhibited significant difference for 3hydroxybenzoic acid and it ranged from 0.89 to $6.06 \mu \mathrm{g} \mathrm{g}^{-1} \mathrm{FW}$ in different genotypes. As evident from treatment means, the maximum 3hydroxybenzoic acid was found in $A$. reticulata $\left(6.06 \mu \mathrm{g} \mathrm{g}^{-1} \mathrm{FW}\right)$ which was followed by $A$. muricata

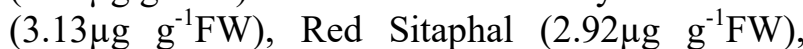
Balanagar $\left(2.89 \mu \mathrm{g} \mathrm{g}^{-1} \mathrm{FW}\right)$ and A. glabra $(1.81 \mu \mathrm{g} \mathrm{g}$ $\left.{ }^{1} \mathrm{FW}\right)$. The minimum3-hydroxybenzoic acid was found in A. cherimola $\left(0.89 \mu \mathrm{g} \mathrm{g}^{-1} \mathrm{FW}\right)$, A. atemoya $\left(1.32 \mu \mathrm{g} \mathrm{g}{ }^{-1} \mathrm{FW}\right)$ and Arka Sahan $\left(1.35 \mu \mathrm{g} \mathrm{g}^{-1} \mathrm{FW}\right)$. The $t$-cinnamic acid was significantly different among the annona genotypes. A range of 3.93 to $22.68 \mu \mathrm{g} \mathrm{g}^{-1} \mathrm{FW}$ of $t$-cinnamic acid was recorded in different genotypes. As evident from the treatment means, maximum $t$-cinnamic acid was found in Arka Sahan $\left(22.68 \mu \mathrm{g} \mathrm{g}^{-1} \mathrm{FW}\right)$ which was followed by Balanagar $\left(18.29 \mu \mathrm{g} \mathrm{g}^{-1} \mathrm{FW}\right)$, Red Sitaphal (17.54 $\left.\mu \mathrm{g} \mathrm{g}^{-1} \mathrm{FW}\right)$, A. atemoya $\left(14.07 \mu \mathrm{g} \mathrm{g}^{-1} \mathrm{FW}\right)$ and A. reticulate $\left(10.20 \mu \mathrm{g} \mathrm{g}^{-1} \mathrm{FW}\right)$. The minimum $t$ cinnamic acid was observed in $A$. cherimola (3.93 $\left.\mu \mathrm{g} \mathrm{g}^{-1} \mathrm{FW}\right)$, A. glabra $\left(3.95 \mu \mathrm{g} \mathrm{g}^{-1} \mathrm{FW}\right)$ and $A$. muricata $\quad\left(4.47 \mu \mathrm{g} \quad \mathrm{g}^{-1} \mathrm{FW}\right)$. The 2,4dihydroxybenzoic acid was significantly different among the annona genotypes. A range of 39.49 to $10.43 \mu \mathrm{g} \mathrm{g}^{-1} \mathrm{FW}$ of 2,4-dihydroxybenzoic acid was recorded in different genotypes. As evident from the treatment means, maximum 2,4dihydroxybenzoic acid was found in $A$. reticulata $\left(39.49 \mu \mathrm{g} \mathrm{g}^{-1} \mathrm{FW}\right)$ which was followed by $A$. glabra (30.87 $\left.\mathrm{g} \mathrm{g} \mathrm{g}^{-1} \mathrm{FW}\right)$, A. muricata $\left(27.84 \mu \mathrm{g} \mathrm{g}^{-1} \mathrm{FW}\right), A$. cherimola (18.38 $\left.\mu \mathrm{g} \mathrm{g}^{-1} \mathrm{FW}\right)$ and Arka Sahan $\left(16.31 \mu \mathrm{g} \mathrm{g} \mathrm{g}^{-1} \mathrm{FW}\right)$. The minimum 2, 4dihydroxybenzoic acid was noticed in A. atemoya $\left(14.83 \mu \mathrm{g} \mathrm{g}^{-1} \mathrm{FW}\right)$, Balanagar $\left(12.39 \mu \mathrm{g} \mathrm{g}^{-1} \mathrm{FW}\right)$ and Red Sitaphal $\left(10.43 \mu \mathrm{g} \mathrm{g}^{-1} \mathrm{FW}\right)$.

Annona genotypes exhibited significant difference for gentisic acid. Gentisic acid ranged from 9.77 to $24.69 \mu \mathrm{g} \mathrm{g}{ }^{-1} \mathrm{FW}$ in different genotypes. The maximum gentisic acid was found in $A$. muricata $\left(24.69 \mu \mathrm{g} \mathrm{g}^{-1} \mathrm{FW}\right)$ which was followed by Balanagar

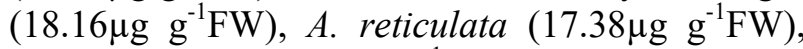
Red Sitaphal $\left(14.84 \mu \mathrm{g} \mathrm{g}^{-1} \mathrm{FW}\right)$ and Arka Sahan
(14.22 $\left.\mu \mathrm{g} \mathrm{g}^{-1} \mathrm{FW}\right)$. The minimum gentisic acid was found in A. atemoya $\left(9.77 \mu \mathrm{g} \mathrm{g}^{-1} \mathrm{FW}\right)$, A. glabra $\left(10.46 \mu \mathrm{g} \mathrm{g}^{-1} \mathrm{FW}\right)$ and $A$. cherimola $(11.82 \mu \mathrm{g} \mathrm{g}$ $\left.{ }^{1} \mathrm{FW}\right)$. The protocatechuic acid was significantly different among the annona genotypes. A range of 4.23 to $17.04 \mu \mathrm{g} \mathrm{g}^{-1} \mathrm{FW}$ of protocatechuic acid was recorded in different genotypes. As evident from the treatment means, maximum protocatechuic acid was found in A. muricata $\left(17.04 \mu \mathrm{g} \mathrm{g}^{-1} \mathrm{FW}\right)$ which was followed by $A$. glabra $\left(16.56 \mu \mathrm{g} \mathrm{g} \mathrm{g}^{-1} \mathrm{FW}\right), A$. cherimola $\left(16.47 \mu \mathrm{g} \mathrm{g}^{-1} \mathrm{FW}\right)$, A. atemoya $(15.82 \mu \mathrm{g}$ $\left.\mathrm{g}^{-1} \mathrm{FW}\right)$ and Arka Sahan $\left(14.33 \mu \mathrm{g} \mathrm{g}^{-1} \mathrm{FW}\right)$. The minimum protocatechuic acid was observed in Balanagar $\left(4.23 \mu \mathrm{g} \mathrm{g}^{-1} \mathrm{FW}\right)$, Red Sitaphal $\left(4.82 \mu \mathrm{g} \mathrm{g}^{-}\right.$ $\left.{ }^{1} \mathrm{FW}\right)$ and $A$. reticulata $\left(6.99 \mu \mathrm{g} \mathrm{g}^{-1} \mathrm{FW}\right)$. There was a significant difference for $p$-coumaric acid among the annona genotypes. A range of 78.54 to $321.53 \mu \mathrm{g} \mathrm{g}^{-1} \mathrm{FW}$ of $p$-coumaric acid was recorded in different genotypes. The maximum $p$-coumaric acid was found in A. muricata $\left(321.53 \mu \mathrm{g} \mathrm{g}^{-1} \mathrm{FW}\right)$ which was followed by $A$. cherimola $\left(260.66 \mu \mathrm{g} \mathrm{g}^{-}\right.$ $\left.{ }^{1} \mathrm{FW}\right)$, A. reticulata $\left(172.65 \mu \mathrm{g} \mathrm{g}^{-1} \mathrm{FW}\right)$, A. glabra

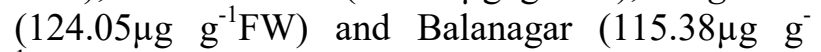
$\left.{ }^{1} \mathrm{FW}\right)$. The minimum $p$-coumaric acid was found in A. atemoya $\left(78.54 \mu \mathrm{g} \mathrm{g}^{-1} \mathrm{FW}\right)$, Arka Sahan $(78.54 \mu \mathrm{g}$ $\left.\mathrm{g}^{-1} \mathrm{FW}\right)$ and Red Sitaphal $\left(103.76 \mu \mathrm{g} \mathrm{g}^{-1} \mathrm{FW}\right)$.

The o-coumaric acid was significantly different among the annona genotypes. A range of 7.91 to $70.80 \mu \mathrm{g} \mathrm{g}^{-1} \mathrm{FW}$ of $o$-coumaric acid was recorded in different genotypes. The maximum $o$-coumaric acid was observed in A. muricata $\left(70.80 \mu \mathrm{g} \mathrm{g}^{-1} \mathrm{FW}\right)$ which was followed by $A$. glabra $\left(50.31 \mu \mathrm{g} \mathrm{g}^{-1} \mathrm{FW}\right)$, A. reticulata $\left(48.64 \mu \mathrm{g} \mathrm{g}^{-1} \mathrm{FW}\right)$, Balanagar $(48.11 \mu \mathrm{g}$ $\left.\mathrm{g}^{-1} \mathrm{FW}\right)$, Red Sitaphal $\left(36.23 \mu \mathrm{g} \quad \mathrm{g}^{-1} \mathrm{FW}\right), \quad A$. cherimola (23.46 $\left.\mu \mathrm{g} \mathrm{g}^{-1} \mathrm{FW}\right)$ and Arka Sahan $\left(19.00 \mu \mathrm{g} \mathrm{g}^{-1} \mathrm{FW}\right)$. The minimum $o$-coumaric acid was found in A. atemoya $\left(7.91 \mu \mathrm{g} \mathrm{g} \mathrm{g}^{-1} \mathrm{FW}\right)$. The perusal of data on vanillic acid indicated significant difference among the annona genotypes. A range of 2.16 to $19.13 \mu \mathrm{g} \mathrm{g}^{-1} \mathrm{FW}$ of vanillic acid was recorded in different genotypes. The maximum vanillic acid was found in A. glabra $\left(19.13 \mu \mathrm{g} \mathrm{\textrm {g } ^ { - }}\right.$ $\left.{ }^{1} \mathrm{FW}\right)$ which was followed by $A$. cherimola $(6.87 \mu \mathrm{g}$ $\left.\mathrm{g}^{-1} \mathrm{FW}\right)$, Balanagar $\left(4.96 \mu \mathrm{g} \mathrm{g}^{-1} \mathrm{FW}\right)$, Red Sitaphal $\left(4.10 \mu \mathrm{g} \mathrm{g}^{-1} \mathrm{FW}\right)$ and $A$. reticulata $\left(3.71 \mu \mathrm{g} \mathrm{g}^{-1} \mathrm{FW}\right)$. The minimum vanillic acid was noticed in $A$. muricata $\left(2.16 \mu \mathrm{g} \mathrm{g}^{-1} \mathrm{FW}\right)$, A. atemoya $(2.59 \mu \mathrm{g} \mathrm{g}$ $\left.{ }^{1} \mathrm{FW}\right)$ and Arka Sahan $\left(3.11 \mu \mathrm{g} \mathrm{g}{ }^{-1} \mathrm{FW}\right)$. There was a significant difference obtained for gallic acid among the annona genotypes and it ranged from 
Polyphenolic profiles in edible Annona spp.

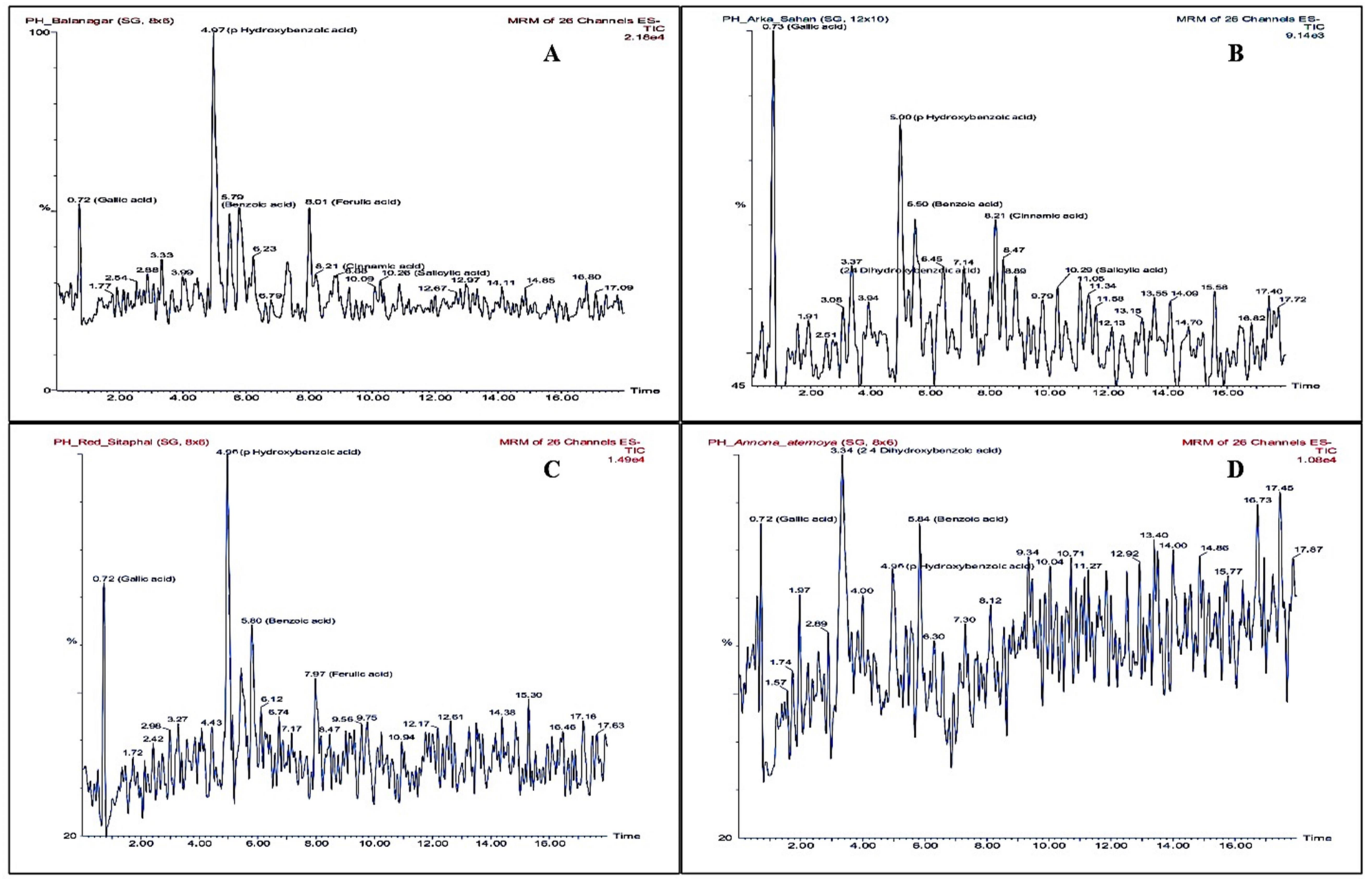

Figure 1a. Chromatograms of phenolic acid profiling in A. squamosa [Balanagar (A), Arka Sahan (B), Red Sitaphal (C)] and A. atemoya (D) 
Shetty et al.

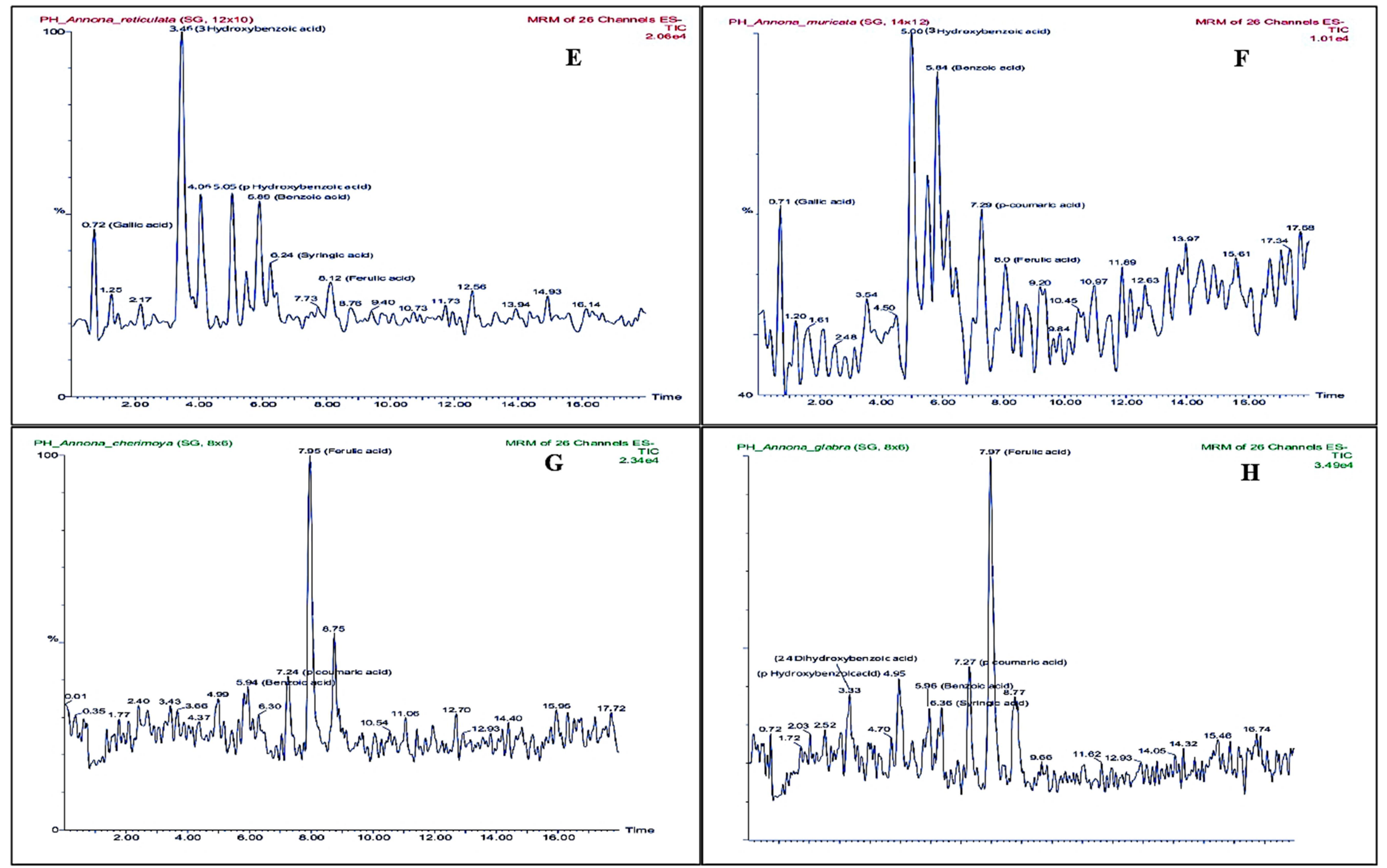

Figure 1b. Chromatograms of phenolic acid profiling in $A$. reticulata (E), A. muricata (F), A. cherimoya (G) and $A$. glabra (H). 
2.74 to $15.88 \mu \mathrm{g} \mathrm{g}^{-1} \mathrm{FW}$. As evident from the treatment means, maximum gallic acid was observed in $A$. reticulata $\left(15.88 \mu \mathrm{g} \mathrm{g}^{-1} \mathrm{FW}\right)$ which was on par with Arka Sahan $\left(14.06 \mu \mathrm{g} \mathrm{g}^{-1} \mathrm{FW}\right)$ and was followed by Balanagar $\left(10.25 \mu \mathrm{g} \mathrm{g}{ }^{-1} \mathrm{FW}\right), A$. atemoya $\left(8.34 \mu \mathrm{g} \mathrm{g}^{-1} \mathrm{FW}\right)$, Red Sitaphal $(6.57 \mu \mathrm{g} \mathrm{g}$ $\left.{ }^{1} \mathrm{FW}\right)$ and $A$. cherimola $\left(6.35 \mu \mathrm{g} \mathrm{g}^{-1} \mathrm{FW}\right)$. The minimum gallic acid was found in A. glabra $\left(2.74 \mu \mathrm{g} \mathrm{g}^{-1} \mathrm{FW}\right)$ and $A$. muricata $\left(6.03 \mu \mathrm{g} \mathrm{g}^{-1} \mathrm{FW}\right)$.

The caffeic acid differed significantly among the annona genotypes and it ranged from 3.43 to 35.26 $\mu \mathrm{g} \mathrm{g}^{-1} \mathrm{FW}$. The maximum caffeic acid was found in A. cherimola $\left(35.26 \mu \mathrm{g} \mathrm{g}^{-1} \mathrm{FW}\right)$ which was followed by $A$. glabra $\left(27.78 \mu \mathrm{g} \mathrm{g}^{-1} \mathrm{FW}\right), A$. reticulata $\left(25.59 \mu \mathrm{g} \mathrm{g}^{-1} \mathrm{FW}\right)$, Arka Sahan $\left(23.88 \mu \mathrm{g} \mathrm{g}^{-1} \mathrm{FW}\right)$ and A. muricata $\left(16.76 \mu \mathrm{g} \mathrm{g}^{-1} \mathrm{FW}\right)$. The minimum caffeic acid was found in A. atemoya $(10.07 \mu \mathrm{g} g$ $\left.{ }^{1} \mathrm{FW}\right)$, Balanagar $\left(6.31 \mu \mathrm{g} \mathrm{g}^{-1} \mathrm{FW}\right)$ and Red Sitaphal $\left(3.43 \mu \mathrm{g} \mathrm{g}^{-1} \mathrm{FW}\right)$. There was a significant difference for ferulic acid among the annona genotypes and it ranged from 5.91 to $21.78 \mu \mathrm{g} \mathrm{g}^{-1} \mathrm{FW}$. The maximum ferulic acid was found in $A$. cherimola $\left(21.78 \mu \mathrm{g} \mathrm{\textrm {g } ^ { - 1 } \mathrm { FW } )}\right.$ which was followed by $A$. muricata $\left(15.94 \mu \mathrm{g} \mathrm{g}^{-1} \mathrm{FW}\right)$, A. glabra $(12.28 \mu \mathrm{g} \mathrm{g}$ $\left.{ }^{1} \mathrm{FW}\right)$, A. atemoya $\left(7.24 \mu \mathrm{g} \mathrm{g}^{-1} \mathrm{FW}\right)$ and Arka Sahan $\left(6.22 \mu \mathrm{g} \mathrm{g}^{-1} \mathrm{FW}\right)$. The minimum ferulic acid was observed in Red Sitaphal (5.91 $\left.\mu \mathrm{g} \mathrm{g} \mathrm{g}^{-1} \mathrm{FW}\right)$, Balanagar $\left(5.93 \mu \mathrm{g} \mathrm{g} \mathrm{g}^{-1} \mathrm{FW}\right)$ and $A$. reticulata $\left(6.13 \mu \mathrm{g} \mathrm{g}^{-1} \mathrm{FW}\right)$. The perusal of data on syringic acid indicated significant difference among the annona genotypes and it ranged from 0.78 to 0.34 $\mu \mathrm{g} \mathrm{g}^{-1} \mathrm{FW}$. As evident from treatment means, the maximum syringic acid was noticed in Red Sitaphal $\left(0.78 \mu \mathrm{g} \mathrm{g}^{-1} \mathrm{FW}\right)$ which was statistically $(p<0.05)$ on par with Balanagar $\left(0.77 \mu \mathrm{g} \mathrm{g}^{-1} \mathrm{FW}\right)$, A atemoya $\left(0.75 \mu \mathrm{g} \mathrm{g}^{-1} \mathrm{FW}\right), A$. cherimola $\left(0.67 \mu \mathrm{g} \mathrm{g}^{-1} \mathrm{FW}\right)$ and A. glabra $\left(0.63 \mu \mathrm{g} \mathrm{g}^{-1} \mathrm{FW}\right)$ and was followed by Arka Sahan $\left(0.34 \mu \mathrm{g} \mathrm{g}^{-1} \mathrm{FW}\right)$, A. muricata $(0.36 \mu \mathrm{g} \mathrm{g}$ $\left.{ }^{1} \mathrm{FW}\right)$ and $A$. reticulata $\left(0.38 \mu \mathrm{g} \mathrm{g}^{-1} \mathrm{FW}\right)$. The sinapic acid differed significantly among the annona genotypes and it ranged from 0.87 to $2.16 \mu \mathrm{g} g$ ${ }^{1} \mathrm{FW}$. The maximum sinapic acid was found in Balanagar $\left(2.16 \mu \mathrm{g} \mathrm{g}^{-1} \mathrm{FW}\right)$ which was followed by A. muricata $\left(1.67 \mu \mathrm{g} \mathrm{g}^{-1} \mathrm{FW}\right)$, A. cherimola $(1.26 \mu \mathrm{g}$ $\left.\mathrm{g}^{-1} \mathrm{FW}\right)$ and Arka Sahan $\left(1.11 \mu \mathrm{g} \mathrm{g}^{-1} \mathrm{FW}\right)$. The minimum sinapic acid was found in $A$. reticulata $\left(0.79 \mu \mathrm{g} \mathrm{g}^{-1} \mathrm{FW}\right)$, A. atemoya $\left(0.86 \mu \mathrm{g} \mathrm{g}^{-1} \mathrm{FW}\right)$, Red Sitaphal $\left(0.87 \mu \mathrm{g} \mathrm{g}^{-1} \mathrm{FW}\right)$ and $A$. glabra $(0.99 \mu \mathrm{g} \mathrm{g}$ $\left.{ }^{1} \mathrm{FW}\right)$. There was a significant difference for ellagic acid among the annona genotypes and it ranged from 0.27 to $1.12 \mu \mathrm{g} \mathrm{g}^{-1} \mathrm{FW}$. The maximum ellagic

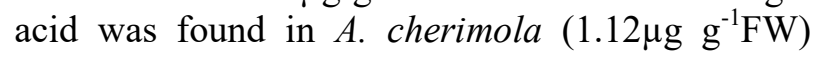
which was followed by $A$. muricata $\left(0.69 \mu \mathrm{g} \mathrm{\textrm {g } ^ { - }}\right.$ $\left.{ }^{1} \mathrm{FW}\right)$, A. reticulata $\left(0.40 \mu \mathrm{g} \mathrm{g}^{-1} \mathrm{FW}\right)$, Balanagar $\left(0.28 \mu \mathrm{g} \mathrm{g}^{-1} \mathrm{FW}\right)$, Arka Sahan $\left(0.27 \mu \mathrm{g} \mathrm{g} \mathrm{g}^{-1} \mathrm{FW}\right), A$. atemoya $\left(0.27 \mu \mathrm{g} \mathrm{g}^{-1} \mathrm{FW}\right)$ and A. glabra $(0.26 \mu \mathrm{g} \mathrm{g}$ $\left.{ }^{1} \mathrm{FW}\right)$. The minimum ellagic acid was observed in Red Sitaphal $\left(0.20 \mu \mathrm{g} \mathrm{g}^{-1} \mathrm{FW}\right)$. Polyphenolic compounds are the secondary metabolites which are widely found in plant and plant derived foods. Several analytical methods are available for detection of the polyphenolics. Most of the times, these polyphenolic compounds are analyzed by High Performance Liquid Chromatography (HPLC) (Giusti et al., 1999; Vagiri et al., 2012), coupled with diode array detector and mass spectrometer (Revilla et al., 1999). In the present investigation, seventeen polyphenolic compounds were identified by comparing their retention times and mass spectra with respective standards. The perusal of mean values indicated that polyphenolic acids viz., $p$ coumaric acid, o-coumaric acid, 2,4dihydroxybenzoic acid, caffeic acid, gentisic acid, protocatechuic acid, $t$-cinnamic acid and ferulic acid were abundant in annona fruits. However, chlorogenic acid was not identified in any of the methanolic extracts of fruits. The custard apple juice was found to contain polyphenolic acids like caffeic, ferulic, $p$-coumaric and sinapic acid in free form (Lee et al., 2003). Cinnamic acid and $p$ coumaric acid were prevalent polyphenolic compounds in A. muricata pulp (Jimenez et al., 2014). $p$-coumaric acid, caffeic acid, protocatechuic acid, gentisic acid and gallic acid were reported in custard apple wine (Jagtap and Bapat, 2015). It is clear from the HPLC-MS/MS analysis that individual polyphenolics were positively correlated with total polyphenolic contents of annona fruits. $A$. muricata was registered with elevated level of polyphenolic compounds, thus have wide health benefits due to their antioxidant characteristics. Reactive oxygen species (ROS) generated during metabolic processes in the human body are eliminated by enzymatic and non-enzymatic antioxidant systems that exist in the body. However, antioxidant system abnormalities, or excess generation of ROS due to various physical and chemical factors, induce oxidative stress, which causes tissue damage and gene mutation, resulting in various chronic diseases, such as diabetes, 
Alzheimer's disease, and aging (Seifried et al., 2007).

\section{Conclusion}

From the above study it may be concluded that research on the development of functional materials from edible natural resources with antioxidant effects is a need of the hour. In the present study, Annona muricata L. has been reported with highest concentration of polyphenolic compounds among the eight Annona spp. fruits. Total eighteen individual compounds were studied in the fruits, among which the $p$-coumaric acid, $o$-coumaric acid, 2,4-dihydroxybenzoic acid, caffeic acid, gentisic acid, protocatechuic acid, $t$-cinnamic acid and

\section{References}

Almeida, M. M. B., de Sousa, P. H. M., Arriage, A. M. C., do Prado, G. M., Magalhaes, C. E. C., Maia, G. A. and Lemos, G. A. 2011. Bioactive compounds and antioxidant activity of fresh exotic fruits from north-eastern Brazil. Food Research International, 44: 2155-2159.

Chatrou, L. W., Pirie, M .D., Erkens, R. H. J., Couvreur, T. L. P., Neubig, K. M., Abbott, J. R., Mols, J. B., Maas, J. W., Saunders, R. M. K. and Chase, M. W., 2012. A new sub familial and tribal classification of the pantropical flowering plant family Annonaceae informed by molecular phylogenetics. Botanical Journal of the Linnean Society, 16(9): 5-40.

Dembitsky, V. M., Poovarodom, S., Leontowicz, H., Leontowicz, M., Vearasilp, S., Trakhtenberg, S. and Gorinstein, S. 2011. The multiple nutrition of some exotic fruits: Biological activity and active metabolites. Food Research International, 44: 1671-1701.

Giusti, M., Rodriguez-Saona, L., Griffin, D. and Wrolstad, R. 1999. Electrospray and tanden mass spectroscopy as tools for anthocyanin characterization. Journal of Agricultural and Food Chemistry, 47(12): 4657-64.

Jagtap, U. B. and Bapat, V. A. 2015. Phenolic composition and antioxidant capacity of wine prepared from custard apple (Annona squamosa L.) fruits. Journal of Food Processing and Preservation, 39(3): 175-182.

Jimenez, V. M., Gruschwitz, M., Schweiggert, R. M., Carle, R. and Esquivel, P. 2014. Identification of phenolic compounds in soursop (Annona muricata) pulp by highperformance liquid chromatography with diode array and electrospray ionization mass spectrometric detection. Food Research International, 65(2): 42-46.

Lee, P. R., Tan, R. M., Yu, B., Curran, P. and Liu, S. Q. 2003. Sugars, organic acids and phenolic acids of exotic ferulic acid. The results obtained in this study will help further the understanding of the polyphenolic composition of Annona fruits and the roles of these compounds in health-promoting physiological functions by nullifying reactive oxygen species (ROS).

\section{Acknowledgment}

Authors are thankful to University of Horticultural Sciences, Bagalkot and Indian Institute of Horticultural Research, Bengaluru for providing the facilities for research. Also sincere gratitude to teaching and non-teaching personnel of College of Horticulture, UHS Campus, GKVK (P), Bengaluru is hereby acknowledged.

seasonable tropical fruits. Nutrition \& Food Science, 43(9): 267-276.

Loizzo, M. R., Tundis, R., Bonesi, M., Menichini, F., Mastellone, V., Avallone, L. and Menichini, F. 2012. Radical scavenging, antioxidant and metal chelating activities of Annona cherimola Mill. (Cherimoya) peel and pulp in relation to their total phenolic and total flavonoid contents. Journal of Food Composition and Analysis, 25: 179-184.

Revilla, I., Magarino, S. P., Gonzalez-SanJose, M. L. and Beltran, S. 1999. Identification of anthocyanin derivatives in grape skin extracts and red wines by liquid chromatography with diode array and mass spectrometric detection. Journal of Chromatography, 847(9): 83-90.

Seifried, H. E., Anderson, D. E., Fisher, E. I. and Milner, J. A., 2007. A review of the interaction among dietary antioxidants and reactive oxygen species. Journal of Nutritional Biochemistry, 18: 567-579.

Sousa, P. M., Elias, S. T., Imenoni, L. A., Paula, J. E., Gomes, S. M. and Guerra, E. N. S. 2012. Plants from Brazilian Cerrado with potent tyrosinase inhibitory activity. Plos One, 7(11): 1-7.

Vagiri, M., Ekholm, A. Andersson, S. C., Johansson, E. and Rumpunen, K. 2012. An optimized method for analysis of phenolic compounds in buds, leaves, and fruits of black currant ( Ribes nigrum L.). Journal of Agricultural and Food Chemistry, 60(7): 105-110.

Weidner, S., Amarowicz, R., Karamac, M. and FrTczek, E. 2000. Changes in endogenous phenolic acids during development of Secale cereale caryopses and after dehydration treatment of unripe rye grains. Plant Physiology and Biochemistry, 38(3): 595-602. 